\title{
Resultados del tratamiento ortopédico del dedo en martillo por ruptura subcutánea del tendón extensor. Serie de 170 casos
}

\author{
C. Muratore ${ }^{(1)}$, J. Medina ${ }^{(2)}$, R. Navarro ${ }^{(2)}$, G. Fouche ${ }^{(2)}$ \\ MÉdico Interno Residente. Hospital Universitario InSUlar GC. \\ ${ }^{(1)}$ UNIVERSIDAD DE LAS PALMAS DE GC. \\ ${ }^{(2)}$ Hospital Universitario Insular de GRan CANaria.
}

Correspondencia:

Dr. Jose A. Medina Henríquez

Luis Doreste Silva, 56

35004 Las Palmas

Tf 606349450

Jmedina-cmano-lp@telefonica.net

Fax: 928232903

Objetivo: El presente estudio tiene por objetivo demostrar que los pacientes con dedo en martillo por arrancamiento del tendón extensor o ruptura subcutánea, pueden beneficiarse del tratamiento ortopédico, reservando el tratamiento quirúrgico para casos con mala evolución.

Material y Método: Se realizó un estudio retrospectivo de un grupo 170 de pacientes que presentaban una deformación digital en martillo por ruptura subcutánea o arrancamiento del tendón extensor. Todos los casos fueron tratados ortopédicamente, ya sea en consulta precoz y hasta en casos con un retraso máximo de 8 semanas. El tratamiento utilizado fue una férula dorsal en forma de rejilla, moldeada a medida, inmovilizando sólo la articulación interfalángica distal en extensión por un período de tiempo medio de 54 días.

Resultados: Con un seguimiento medio de 5 meses, el déficit de extensión fue de $7^{\circ}$ y la flexión alcanzó los $61^{\circ}$. Setenta y ocho pacientes de este grupo fueron evaluados después de un plazo medio de 5 años, obteniendo similares resultados, con un déficit de extensión medio de $5^{\circ}$ y una movilidad en flexión media de $61^{\circ}$.
Objective: The objective of the present study is to demonstrate that patients with mallet finger by pulling up of the extensor tendon or subcutaneous rupture, can benefit from the orthopaedic treatment, leaving surgical treatment for cases with bad evolution.

Material and Method: A retrospective study was carried out in 170 patients who presented a digital deformation in mallet finger by subcutaneous rupture or pulling up of the extensor tendon. All cases were treated nonsurgically, even cases with a maximum delay of 8 weeks. The treatment used was a dorsal custom-made perforated splint, immobilizing only the distal interphalangeal joint (DIP) in extension for an average period of 54 days.

Results: After an average follow-up of 5 months, the extension deficit was of $7^{\circ}$ and the flexion reached $61^{\circ}$. Seventy-eight patients of this group were evaluated after an average follow-up of 5 years, having obtained similar results, with an average deficit of extension of $5^{\circ}$ and an average flexion of $61^{\circ}$.

Conclusion: The splint is the elected treatment even in cases with delay of presentation; surgery is only 
Conclusiones: La férula es pues el tratamiento de elección incluso en caso de consulta diferida; la cirugía sólo la utilizamos para casos de fracaso del tratamiento ortopédico y en pacientes exigentes ya sea por razones profesionales o de ocio.

Palabras clave: tendón extensor, dedo en martillo indicated in cases of failure of the orthopaedic treatment, in demanding patients, for professional reasons or for leisure.

Key words: extensor tendon, mallet finger.

Rev. Iberam. Cir. Mano - Vol. 34 • Núm. 1 • Abril 2006 (31-37)

\section{INTRODUCCIÓN}

$\checkmark 1$ 1 dedo en martillo (DM) es una deformación frecuente de la cadena digital, caracterizada por la caída en flexión de la falange distal debida a un arrancamiento óseo o a la ruptura parcial o total del tendón extensor terminal de los dedos. El tratamiento ortopédico se utiliza para los dedos en martillo vistos pronto después del traumatismo mientras que, el tratamiento quirúrgico, sólo se indica para los fracasos del tratamiento conservador y

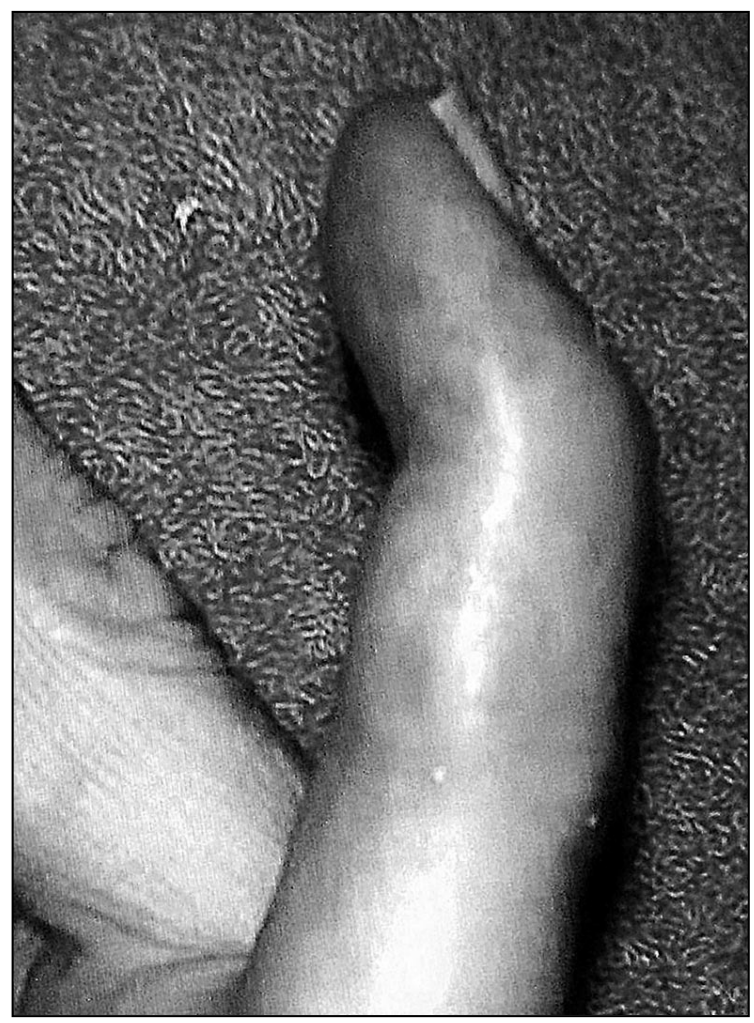

Figura: Mallet finger de $5^{o}$. los DM vistos tardíamente, en fase crónica con una interfalángica distal flexible o con una deformación en cuello de cisne.

El límite, según la literatura entre casos agudos y crónicos es bastante dispar, oscilando entre 3 y 4 semanas. Por nuestra parte, pasado este límite, seguimos utilizando el tratamiento ortopédico hasta el segundo mes.

Revisamos 170 dedos en martillo consecutivos tratados ortopédicamente, excluyendo los traumatismos abiertos y las fracturas articulares que abarcan mas de $1 / 3$ de la superficie.

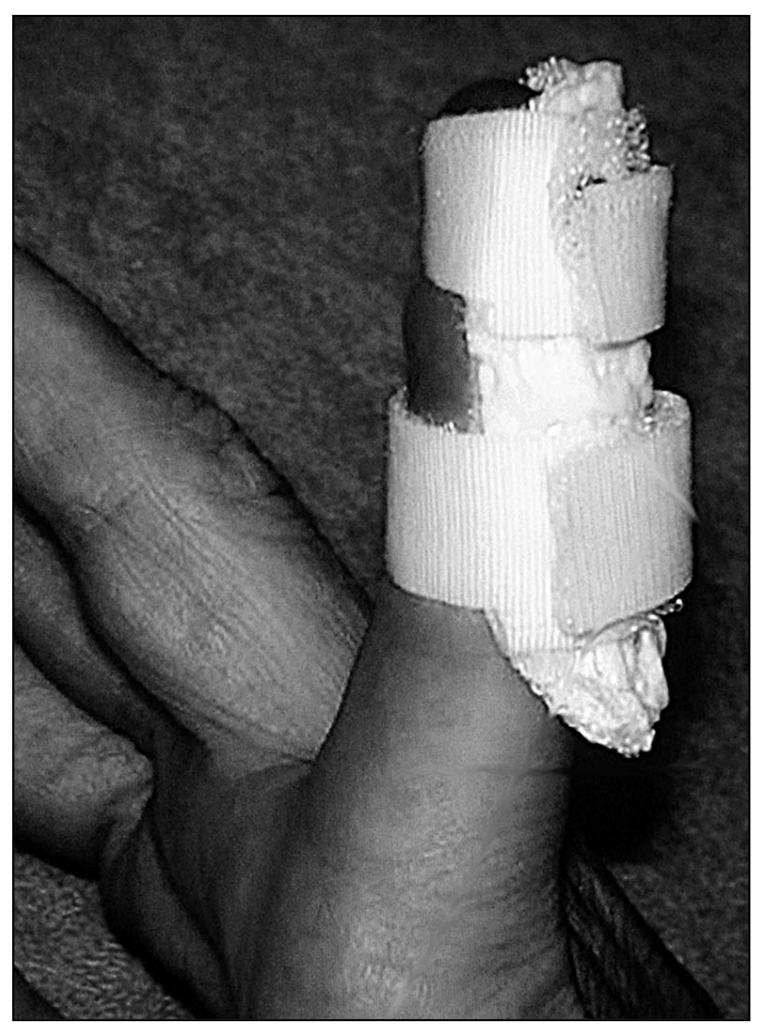

Figura 2: Férula dorsal en $5^{o}$ dedo. 
Tabla I - LOCALIZACIÓN DEL DEDO EN MARTILLO

\begin{tabular}{|llc|}
\hline pulgar & 14 & $7 \%$ \\
\hline indice & 10 & $4 \%$ \\
\hline medio & $\mathbf{6 2}$ & $\mathbf{3 7 \%}$ \\
\hline anular & 42 & $26 \%$ \\
\hline Auricular & 42 & $26 \%$ \\
\hline
\end{tabular}

Tabla II - EtıologíA

\begin{tabular}{|lcc|}
\hline Accidente laboral & 31 & $21 \%$ \\
\hline Accidente doméstico & $\mathbf{5 2}$ & $\mathbf{3 4 \%}$ \\
\hline Deporte & 49 & $33 \%$ \\
\hline Caídas & 12 & $7 \%$ \\
\hline Otros & 10 & $5 \%$ \\
\hline Total & 154 & \\
\hline (desconocidos: 16) & \\
\hline
\end{tabular}

\section{MATERIAL Y MÉTODO}

El tratamiento conservador consistió en una inmovilización con una férula dorsal en material termoformado [X-Lite (R)], moldeada a medida, sólo inmovilizando la articulación interfalángica distal con una muy discreta hiperextensión. La fijación se aseguró con velcro, duplicado con una fina banda de elastoplast con el fin de evitar todo deslizamiento de la férula (en particular, en la flexión IFP) (Figuras 1 y 2).

La duración del tratamiento fue de 7 a 8 semanas, y se autorizó la flexión interfalángica proximal enseñando al paciente que debe evitarse toda flexión distal (por contracción aislada del flexores los superficiales).

Se efectuó el primer cambio de férula al $8^{\circ}$ día, el paciente realizó una semana más tarde su primer cambio bajo el control y los cambios posteriores se confiaron al propio paciente sin otro control. Al final del segundo mes, la férula se retiraba durante el día aunque se aconse- jó su uso nocturno por 2 a 3 semanas mas, plazo al final del cual se revisaron a los pacientes. En caso de déficit de flexión, ninguna férula para recuperarla se indicó antes del tercer mes. La mayor parte del tiempo la reincorporación laboral se reanudó entre la primera y la tercera semana siguiendo el mismo tipo de actividad profesional previamente desarrollada.

El grupo de pacientes tratados incluyó a 75 mujeres y a 95 hombres con una edad media de 42 años en ambos sexos. El déficit de extensión inicial medio era de $35^{\circ}$. El plazo entre el accidente y la primera consulta fue por término medio de 22 días. Los detalles de la localización aparecen en la Tabla I, que muestra la mayor frecuencia de casos de lesión en el $3^{\text {o }}$ dedo $(37 \%)$.

La mano derecha fue la mas afectada con un $59 \%$ de los casos. El accidente doméstico fue la etiología más frecuente (34\%), seguido del accidente deportivo (33\%) (Tabla II).

Los traumatismos abiertos se excluyeron de la serie, así como las fracturas articulares de la IFD mayores de $1 / 3$ de superficie. Un arrancamiento óseo estuvo presente en un $6 \%$ de las observaciones.

\section{RESULTADOS}

La duración media del uso de la férula fue de 54 días. No hubo ninguna complicación cutánea fuera de 3 casos de simple maceración $\sin$ necrosis .

Evaluado después de un plazo medio de 154 días, el déficit medio de extensión había disminuido de $35^{\circ}$ a $7^{\circ}$ y la flexión, determinada por un observador independiente alcanzó los $61^{\circ}$.

Setenta y ocho de estos pacientes se evaluaron nuevamente después de un plazo medio de 61 meses. En dicha fecha, el déficit medio de extensión fue de $5^{\circ}$ y la flexión de $61^{\circ}$ (Figura 3). Para el estudio estadístico de estos resultados utilizamos un test de comparación de medias para muestras repetidas, no existiendo diferencia estadísticamente significativa en los resultados a medio y largo plazo.

Un único paciente presentó en esta fase dolores residuales y 6 referían una persistente intolerancia al frío. 


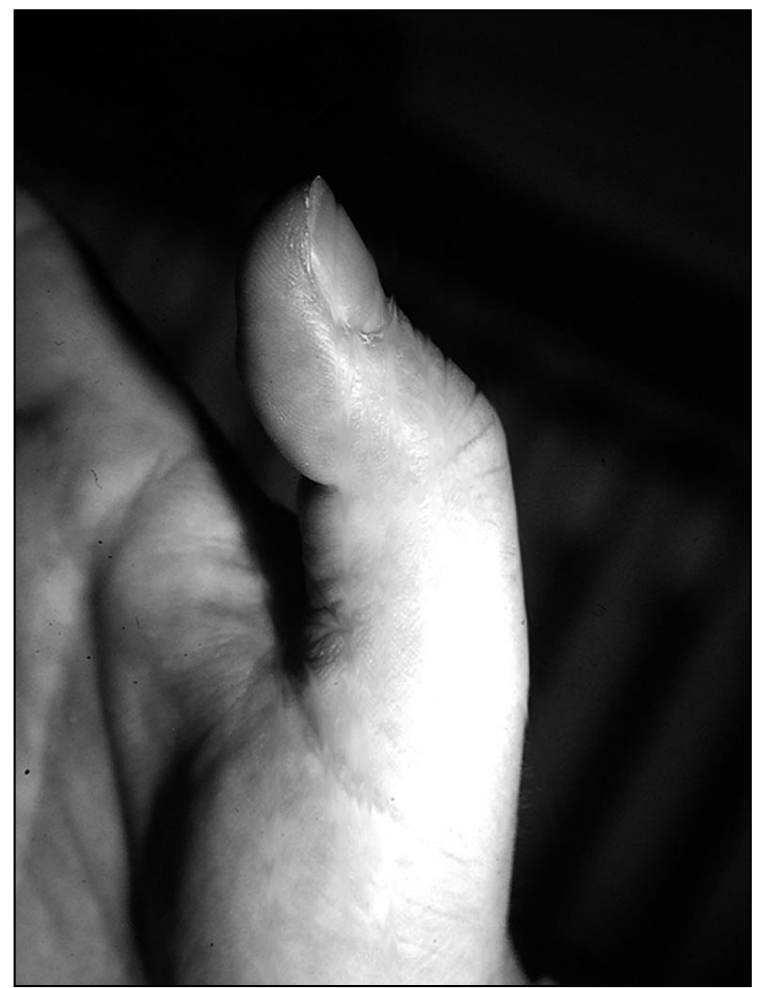

Figura 3: Mallet finger en pulgar.

También se utilizó como metodo estadístico el análisis por regresión, el mismo no permitió poner de relieve correlación entre este déficit de extensión y factores como la edad, el sexo, el segmento alcanzado, el retraso del tratamiento o la presencia de un arrancamiento óseo.

\section{DISCUSION}

Existe consenso en la literatura sobre la eficacia del tratamiento ortopédico del dedo en martillo reciente. Asimismo, numerosas publicaciones describen la eficacia del tratamiento conservador en caso de retraso del tratamiento $^{6,5,7,26,29,12}$ lo que corrobora nuestro estudio (retraso medio de 22 días con un máximo de 58 días).

Más controvertidos permanecen el tipo de férula utilizado, la duración del tratamiento, la mejora espontánea a largo plazo y los resultados.

A partir de 1937, Smilie ${ }^{32}$ proponía el tratamiento con yeso moldeado, manteniendo la IFD en hiperextensión y la IFP en flexión, con el fin

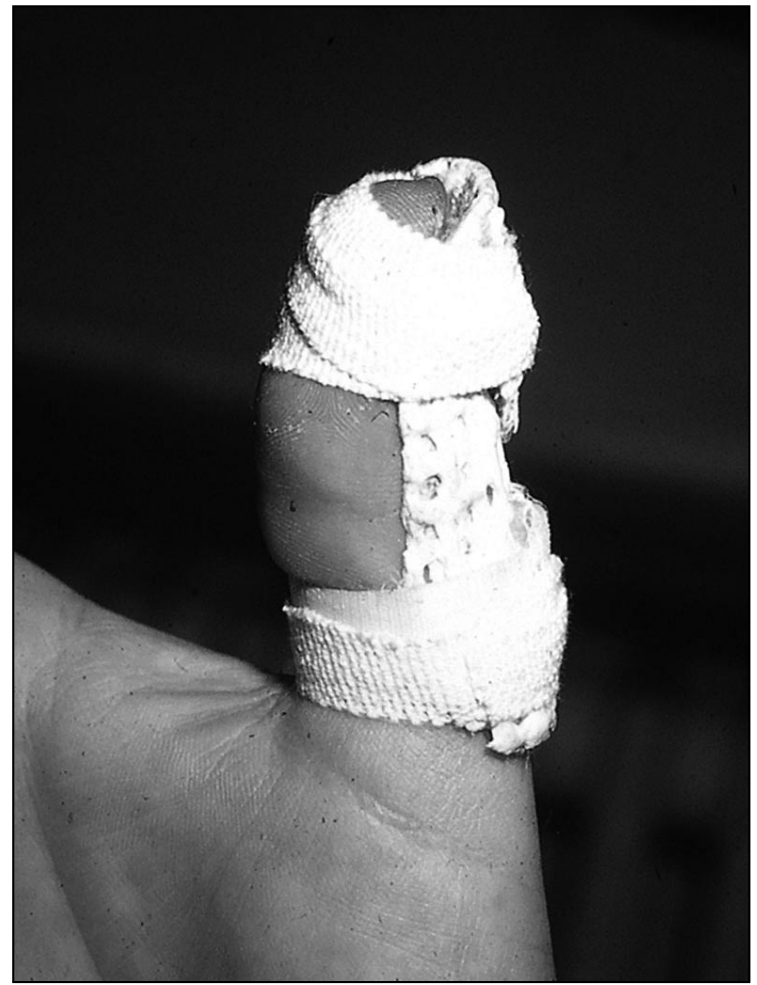

Figura 4: Férula dorsal de primer dedo.

de relajar las bandeletas laterales. Su adaptación no era fácil ya que, si estaba muy ajustada, aumentaba el riesgo de necrosis del pulpejo. Pratt ${ }^{27}$ sustituía el yeso por una aguja axial que garantizaba esta misma posición, evitaba la complicación antes citada pero subsistía a menudo un flexo residual de la IFP (a menudo aumentado por la transfixión del tendón flexor) y aceptaba por otro lado el riesgo infeccioso de la aguja que no tenía buena protección. En realidad, fue Littler ${ }^{21}$ quien puso de manifiesto que el tendón terminal no se retrae y permanece in situ. Era pues lógico no inmovilizar más que la articulación IFD ${ }^{18}$. Por el contrario, la hiperextensión aumenta el riesgo de necrosis dorsal $^{14}$, predecible por el blanqueo cutáneo ${ }^{28}$. En 1969 , Stack ${ }^{35}$ presentó su férula corta, mejorada en $1986^{36}$, disminuyendo la maceración y liberando un poco el pulpejo. Posteriormente se desarrollaron un gran número de mode$\operatorname{los}^{10}, 11,4,23,33,34,1,24,8,16,9,15,30,43,20$. Nosotros nos basamos en la descripción de Elliot $^{8}$ para la localización dorsal de la férula, liberando completamente el pulpejo. 


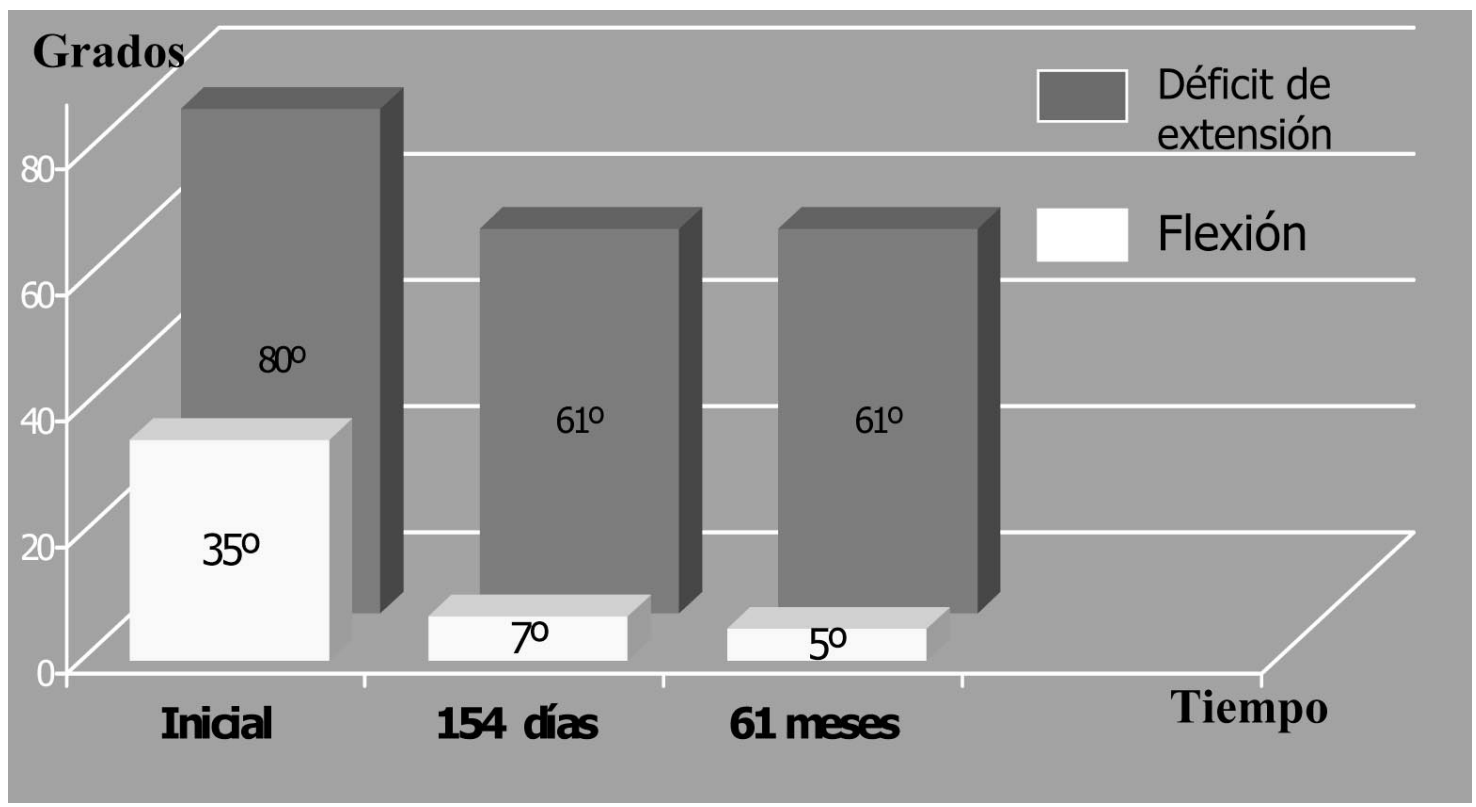

Figura 5: Resultados de grados de flexión y déficit de extensión en sucesivos controles.

Elegimos un material termoformado ventilado. Kinninmonth ${ }^{19}$,comparando dos series de pacientes, demostró la superioridad de las férulas perforadas en cuanto a la higiene, la tolerancia cutánea y la disminución de las complicaciones que están vinculadas. La simplicidad de la férula no exime de una muy cuidadosa educación del paciente para asegurarse de su colaboración. Pensamos que eso nos permitió evitar en nuestra serie toda complicación cutánea importante y es un punto que es necesario no descuidar puesto que Stern ${ }^{38}$ observó un porcentaje de $45 \%$ de complicaciones, esencialmente cutáneas, en su serie de pacientes tratados con férula. Esta férula nos pareció capaz de reunir las cualidades exigibles de simplicidad, ligereza, comodidad, eficacia, precio reducido e inocuidad (para la piel y la articulación proximal). Su sola contraindicación sigue siendo la ausencia de cooperación del paciente. No tenemos experiencia en la utilización de una «ferulización interna» con fijación transarticular oculta de la $\mathrm{IFD}^{2}$. Sin embargo tuvimos ocasión de ver de forma secundaria a 3 pacientes tratados según este método con una aguja doblada de extracción difícil y 2 infecciones sobre el punto de exteriorización de la aguja. Por otra parte, el estudio comparativo de Auchincloss no pudo demostrar superioridad de este método sobre la férula externa ${ }^{2}$.

La duración del tratamiento es también difícil de fijar en función de datos científicos. Según la literatura, la duración varía de $3^{42}$ y 4 semanas $^{31}$ a 9 semanas $^{37}$. Los únicos argumentos válidos para nosotros provienen de Abouna ${ }^{1}$ que habría observado mejores resultados con una inmovilización más larga. Esto coincide con la aparición de recidivas después de extracciones precoces de la férula ${ }^{3}$.

Por otra parte, no fuimos capaces de aislar factores que influían sobre el pronóstico como la edad ${ }^{1}$, la importancia del déficit antes del tratamiento ${ }^{1}$, la localización digital o el retraso del tratamiento.

A nuestro modo de ver, las indicaciones quirúrgicas siguen siendo raras por dos razones importantes: la buena tolerancia a medio plazo de la deformación con desventaja más estética que funcional y la eficacia del tratamiento ortopédico $^{17,13}$. Una hipotética tercera razón sería la posibilidad de mejora espontánea ${ }^{39,40,41}$. En su serie, Warren lo tuvo en cuenta en 13 de los 30 casos que habían rechazado una intervención 
correctora $^{41}$.El estudio sistemático de un grupo de pacientes a medio y largo plazo no nos permitió confirmar este concepto.

\section{CONCLUSIONES}

Así , en conclusión, el tratamiento con férula perforada dorsal, sólo inmovilizando la ar- ticulación interfalángica distal por un período de 8 semanas nos parece un método válido para el tratamiento del dedo en martillo, incluso después de un retraso de tratamiento próximo a dos meses.

Por el contrario, la insuficiencia del resultado puede recuperarse, en un paciente exigente pero cooperador, al precio de un posterior tratamiento quirúrgico.
1. Abouna JM, Brown HG. The treatment of Mallet Finger. $\mathrm{Br} \mathrm{J}$. Surg., 1968, 55, 653-667.

2. Auchincloss JM. Mallet-finger injuries: a prospective, controlled trial of internal and external splintage. Hand, 1982, 14, 2, 168-173.

3. Bendre AA, Hartigan BJ, Kalainov DM. Mallet finger. J Am Acad. Orthop Surg. 2005 Sep; 13(5): 336-44.

4. Brooks D. Splint for Mallet Finger. Br. Med. J., 1964, 1, 1238.

5. Casscells SW, Strange TB. Intramedullary Wire Fixation of Mallet Finger. J. Bone Joint Surg., 1957, 39A, 521-526.

6. Crawford GP. The molded polythene splint for mallet finger deformities. J. Hand Surg., 1984, 9A, 2, 231-237.

7. Elbaz JS, Darcy M, Reynier J, Garnier H. La rupture sous-cutanée distale des tendons extenseurs des doigts. Ann. Chir., 1965, 19, 9-10, 676-679.

8. Elliot Jr RA. Splints for mallet and boutonniere deformities. Plast. Reconstr. Surg., 1973.

9. Evans D, Weightman B. The pipflex splint for treatment of mallet finger. J. Hand Surg., 1988, 13B, 2, 156-158.

10. Flinchum D. Mallet Finger. J. Med. Assn. Georgia, 1959, 48, 601-603.

11. Fowler FD. New Splint for Treatment of Mallet Finger. J. Am. Med. Assn., 1959, 170, 945.

12. Garberman SF, Diao E, Peimer CA. Mallet finger: results of early versus delayed closed

\section{BIBLIOGRAFÍA}

treatment. J Hand Surg [Am] 1994 Sep;19(5):850-2.

13. Geyman JP, Fink K, Sullivan SD Conservative versus surgical treatment of mallet finger: a pooled quantitative literature evaluation. J Am Board Fam Pract. 1998 Sep-Oct; 11(5): 382-90.

14. Hanna F, Tuchmann L. Complication inattendue de l'embrochage en hyper-extension des doigts. Nouv. Presse Méd. 1975, 4, 37.

15. Hart RG, Kleinert HE, Lyons K. The Kleinert modified dorsal finger splint for mallet finger fracture. Am J Emerg Med. 2005 Mar; 23(2): 145-8.

16. Hovgaard C., Klareskov B. Alternative conservative treatment of mallet-finger injuries by elastic double-finger bandage. J. Hand Surg., 1988, 13B, 2, 154-155.

17. Kalainov DM, Hoepfner PE, Hartigan BJ, Carroll C. 4th, Genuario J. Nonsurgical treatment of closed mallet finger fractures. J Hand Surg [Am]. 2005 May;30(3):580-6.

18. Katzman BM, Klein DM, Mesa J, Geller J, Caligiuri A.: Immobilization of the mallet finger. Effects on the extensor tendon.. J Hand Surg [Br]. 1999 Feb; 24(1): 80-4.

19. Kinninmonth WG, Holburn F. A Comparative Controlled Trial of a New Perforated Splint and a Traditional Splint in the Treatment of Mallet Finger. J. Hand Surg., 1986, 11B, 2, 261-262.
20. Lester B, Jeong GK, Perry D, Spero L. A simple effective splinting technique for the mallet finger. Am J Orthop. 2000 Mar; 29(3): 202-6.

21. Littler JW. Commentary on «A new method of treatment for mallet finger» by $\mathrm{H}$. Weinberg et al. Plast. Reconstr. Surg., 1976, 58, 4, 499-500.

22. Lucas GL. Fowler Central Slip Tenotomy for Old Mallet Deformity. 1987, 80, 1, 92-94.

23. Mason ML. Mallet Finger. Lancet, 1954, 1, 1220.

24. Mikic Z, Helal B. The treatment of the mallet finger by Oakley splint. Hand, 1974, 6, 1, 7680.

25. Okafor B, Mbubaegbu C, Munshi I, Williams DJ. Mallet deformity of the finger. Fiveyear follow-up of conservative treatment. J Bone Joint Surg Br. 1997 Jul; 79(4): 544-7.

26. Patel MR, Desai SS, BassiniLipson L. Conservative management of chronic mallet finger. J. Hand Surg., 1986, 11A, 4, 570-573.

27. Pratt DR. Internal splint for closed and open treatment of injuries of. the extensor tendon at the distal joint of the finger. J. Bone Joint Surg., 1964, 46A, 4, 900-903.

28. Rayan GM, Taylor Mullins P. Skin necrosis complicating mallet finger splinting and vascularity of the distal interphalangeal joint overlying. skin. J. Hand Surg., 1987, 12A, 4, 548551. 
29. Reiter A, Hasan M, Unglaub F, Dreyhaupt J, Hahn P. Conservative treatment results of the acute and chronic mallet finger. Unfallchirurg. 2005 Jul 21.

30. Richards SD, Kumar G, Booth S, Naqui SZ, Murali SR. A model for the conservative management of mallet finger. J Hand Surg [Br]. 2004 Feb; 29(1): 61-3.

31. Robb WAT. The results of treatment of mallet finger. J. Bone Joint Surg., 1959, 41B, 3, 546-549.

32. Smilie IS. Mallet Finger. Br. J. Surg., 1973, 24, 439-445.

33. Snow SS. Constructing an improved splint for mallet finger deformity. Plast. Reconstr. Surg., 1973, 52, 5, 586-588.
34. Spigelman L. New Splint for Management of Mallet Finger. J. Am. Med. Assn., 1953, 153, 1362.

35. Stack HG. Mallet Finger. Hand, 1969, 1, 2, 83-89.

36. Stack HG. A Modified Splint for Mallet Finger. J. Hand Surg., 1986, 11B, 2, 263.

37. Stack HH, Boyes JH, Wilson JN. Mallet finger. J. Bone Joint Surg., 1962, 44A, 1061-1068.

38. Stern PJ, Kastrup JJ. Complications and prognosis of treatment of mallet finger. J. Hand Surg., 1988, 13A, 3, 329-334.

39. Vilan R, Dupuis JF. Traitement des ruptures distales du tendon extenseur des doigts (sur 26 cas). Presse Méd., 1971, 79, 44, 1957-1959.
40. Warren RA, Norris SH, Ferguson DG. Mallet finger: a trial of two splints. J. Hand Surg., 1088, 13B, 2, 151-153.

41. Warren RA, Kay NR, Ferguson DG. Mallet finger: comparison between operative and conservative management in those cases failing to be cured by splintage. J. Hand Surg., 1988, 13B, 2, 159-160.

42. Weinberg $\mathrm{H}$, Stein $\mathrm{HC}$, Wexler M. A new method of treatment for mallet finger. Plast. Reconstr. Surg., 1976, 58, 4, 347349.

43. Wilson SW, Khoo CT. The Mexican hat splint-a new splint for the treatment of closed mallet finger. J Hand Surg [Br]. 2001 Oct; 26(5): 488-9. 